\title{
Aproximación a la investigación psicológica en Internet y redes sociales
}

\section{Internet and Social Networks Sites in Psychology Researches: An Approach}

\author{
Vanessa Pérez \\ Universidad Rey Juan Carlos, España \\ (Rec.: septiembre de 2017 - Acep.: enero de 2018)
}

\begin{abstract}
Resumen
El objetivo de este artículo es presentar un resumen de las principales líneas de investigación psicológica en internet y las redes sociales. Dentro de las áreas de investigación más frecuentes se encuentran la construcción de la identidad online, las relaciones personales en internet, el apoyo social online y el bienestar psicológico. Las personas utilizan las redes sociales como una forma de autopresentación y autopromoción, en la que suelen destacar los rasgos narcisistas al desear ser percibidos de manera positiva por los demás. También, se han convertido en espacios de interrelación en los que se sostienen vínculos de amistad, familiares, de pareja y de relaciones grupales en general. Las investigaciones sostienen que las redes sociales aumentan la percepción de capital y apoyo social y contribuyen de manera positiva al bienestar psicológico. Actualmente, las oportunidades de investigación están en el big data y las aplicaciones informáticas para el análisis y la intervención en psicología.
\end{abstract}

Palabras claves: Redes sociales, internet, psicología, investigación.

\begin{abstract}
The aim of this paper is to describe the main topics of psychological research on the internet and social networks. The most frequent research topics are related with online identity construction, personal relationships on the internet, online social support and psychological well-being. People use social networks as a way of self-presentation and self-promotion, in which narcissistic traits often emphasize their desire to be perceived positively by others. Also, social networks on internet have become personal interrelation spaces of friendship, family, couple and group relations in general. Researchers suggest that social networks increase the perception of social capital, social support and contribute positively to psychological well-being. Currently, the new research opportunities are connected with big data and mobile app for analysis and intervention in psychology.
\end{abstract}

Keywords: Social networking sites, internet, psychology, research.

\footnotetext{
* Correspondencia: Departamento de Psicología, Campus de Alcorcón, Madrid, España. Correo electrónico: vanesa. perez@urjc.es
} 


\section{Introducción}

Internet y las redes sociales son una de las áreas y herramientas de investigación psicológica más importantes de las últimas dos décadas (Gosling \& Mason, 2014; Sargis, Skitka \& McKeever, 2013; Wolfe, 2017). Al utilizarse como herramienta de investigación, internet conlleva ventajas como el aumento de la eficacia, la posibilidad de acceder a personas con características especiales, la utilización de muestras más grandes y variadas a menor coste y el incremento de la calidad de los datos (Gosling \& Mason, 2014; Skitka \& Sargis, 2005; Wolfe, 2017). Este tipo de investigaciones favorece el ahorro de tiempo y dinero en la recogida de los datos, ya que permiten evitar los costes asociados a los cuestionarios de papel y la distribución en la población; además, el tiempo requerido para la recolección de los datos es independiente del tamaño de la muestra (Gosling \& Mason, 2014; Skitka \& Sargis, 2005; Wolf, 2017). También permiten el acceso a personas con "características de baja incidencia en la población general" (Skitka \& Sargis, 2005, p. 9) como por ejemplo, personas con discapacidad o grupos violentos. La muestra, en este tipo de investigaciones, suele ser amplia, diversa y accesible en la mayoría de los casos, y asimismo, internet permite la utilización de otros estímulos para los participantes en forma de vídeos, imágenes y textos (Gosling \& Mason, 2014; Skitka \& Sargis, 2005). Otra ventaja a destacar, es la posibilidad de "licencia creativa" en el uso de diversas metodologías de investigación (Sitka \& Sargis, 2006). Por ejemplo, realizar un muestreo de las bibliotecas de música online, estudios sobre páginas web, usar representaciones gráficas de los perfiles de personalidad de los usuarios, entre otros (Sitka \& Sargis, 2006). Dentro de las desventajas de la investigación psicológica, se consideraba que los usuarios de internet diferían de los no usuarios y por tanto podrían producirse algunos sesgos en la selección de la muestra (Sitka \& Sargis, 2006). No obstante, debido al amplio uso de las redes por parte de la población mundial actual, este sesgo estaría disminuido (Gosling \& Mason, 2014). Otras limitaciones incluirían la predisposición positiva de los participantes a responder afirmativamente a invitaciones de investigación en internet, aunque no lo hagan en otro tipo de investigaciones: el anonimato y la baja responsabilidad de las acciones en la web y la imposibilidad de controlar los aspectos contextuales de los participantes (Skitka \& Sargis, 2005). Así mismo, debido a que cada vez es más difusa la línea entre lo que se hace online y lo que se hace offline, este tipo de limitaciones cada vez son menos frecuentes (Gosling \& Mason, 2014; Wolfe, 2017). Por otro lado, se ha comprobado que los datos recogidos online y recogidos de manera tradicional no difieren significativamente (Casler, Bickel \& Hackett, 2013).

Otra desventaja a considerar es lo que algunos autores denominan "limitación ética" (Gosling \& Mason, 2014; Sitka \& Sargis, 2006). Teniendo en cuenta que muchos datos publicados en internet son de acceso libre o de dominio público, algunos investigadores podrían pensar que no es necesario informar a los participantes de la investigación o solicitar su consentimiento informado, lo que puede ser interpretado como una "violación de la privacidad" (Sitka \& Sargis, 2006, p. 549). Además, el hecho de solicitar el consentimiento puede traer algunos problemas al investigador ya que no siempre es posible confirmar la identidad de las personas en la red, los participantes pueden interrumpir de manera inmediata su participación en la investigación al solicitársele el consentimiento, y tampoco el investigador puede asegurar -en todos los casos- que la persona leyó el documento informativo (Sitka \& Sargis, 2006). En la actualidad se considera que este sigue siendo un problema a resolver en los estudios realizados en internet, ya que los consentimientos informados deben ser diseñados específicamente para su uso online. Esto implica que sea necesario solicitar una respuesta o feedback de los participantes que garantice que han comprendido adecuadamente las razones del estudio (para una revisión más detallada sobre este tema ver Kosinski, Matz, Gosling, Vesselin \& Stitwell, 2015), además de tener en cuenta las consideraciones legales de cada país (Kosinski et al., 2015; Lazar, Feng \& Hochheiser, 2010).

Existen tres categorías para clasificar los estudios que utilizan internet y las redes sociales: 1) una aproximación "traslacional", es decir adaptar el material de investigación tradicional $\mathrm{u}$ offline para usarlo en internet, por ejemplo: cuestionarios de papel y lápiz validados en investigaciones tradicionales; 2) una aproximación novedosa u original al utilizar internet como un nuevo método de recoger información o datos para la investigación, por ejemplo: usar la información disponible de manera abierta por las personas en internet (preferencias musicales, foros de discusión, subastas online, etc.); y 3) una aproximación fenomenológica que considera que la dinámica de interacción social en la web es un foco de interés en sí mismo (Gosling \& Mason, 2014; Skitka \& Sargis, 2005). Como señalan Skitka y Sargis, (2005) "investigan para explorar el impacto (...) que tiene la interacción social basada en la web en los pensamientos, sentimientos y comportamientos de las personas" (p. 5). Utilizando esta clasificación Skitka y Sargis (2005) realizaron una revisión de los estudios publicados en psicología durante los años 2003 y 2004 y actualizaron esta información al año 2009 y 2010 (Sargis et al., 2013). El 65\% de los estudios revisados en 2009 y 2010 se habían realizado completamente de manera online lo que muestra el incremento significativo de este tipo de estudios con respecto a la revisión del año 2003 y 2004 en el que representaba solo un 1\% (Sargis et al., 2013). Además, este tipo de investigaciones no solo han sido publicadas en las principales revistas sino que han contribuido al surgimiento de journals especializados como Cyberpsychology, Behavior, and Social Networking, o Journal of Media Psychology, entre otros (Sargis et al., 2013).

Actualmente, la tipología fenomenológica es la que más se utiliza en investigación, ya que internet ha creado nuevas formas de interacción a través de las redes sociales como Instagram, Facebook o Twitter (Gosling \& Mason, 2014). Los efectos que tienen estos escenarios en el comportamiento de las personas se han estudiado en investigaciones sobre la construcción de la identidad online, las relaciones personales en internet, el apoyo social online y el bienestar psicológico, entre otros.

El objetivo de este artículo es presentar un resumen de las principales líneas de investigación psicológica en internet y las redes sociales, desde esta perspectiva más fenomenológica.

Investigaciones sobre la Construcción de la Identidad en Internet y las Redes Sociales

El impacto que tiene el uso de internet en la construcción de la identidad es un tema investigado a partir del desarrollo que tuvieron los espacios anónimos de comunicación entre per- 
sonas como los chats y los foros (Mehdizadeh, 2010; Zhao, Grasmuck \& Martin, 2008). Estas primeras investigaciones centraron su atención en la forma como las personas modificaban su identidad aprovechándose del anonimato en algunos espacios virtuales y la construcción de la identidad a partir de la utilización de pocas señales sociales (pues el tono de voz, la expresión facial, o el aspecto físico, no eran posibles en ese momento de desarrollo tecnológico) (Zhao et al., 2008).

Así, un estudio realizado por Zhao et al. (2008) exploró la construcción de la identidad en Facebook aprovechando las características de esta red social (identificación del usuario a partir del nombre y foto, información sobre gustos, aficiones, comentarios en el muro, etc.). Los autores esperaban que los usuarios de esta red social utilizaran estrategias de autopresentación relacionadas más con lo que les gustaría ser (yo ideal) que con lo que realmente eran (yo real). En este sentido, los usuarios deberían mostrar a sus "amigos" de la red aquella información socialmente deseable y ocultar aquella que no resultaba tan atractiva o favorable para ellos (Zhao et al., 2008). Luego de analizar 83 perfiles de esta red social los resultados mostraron que los usuarios utilizaban diferentes estrategias en la construcción de su identidad social en Facebook denominadas por los autores: implícita (self como actor social) y explícita (self en primera personal). En la estrategia implícita los usuarios reflejaban en su perfil de Facebook información visual, principalmente a partir de fotos y de los comentarios de sus "amigos" en el muro. En el caso de la estrategia explícita los usuarios incluían información verbal sobre quiénes eran, utilizando la herramienta "acerca de mí" que incluye Facebook. La mayoría de los usuarios utilizaba una estrategia implícita, además de presentar más información relacionada con su yo ideal. Por tanto, los autores concluyeron sobre la importancia del contexto en la construcción social de la identidad y sobre cómo el uso de espacios que identifican a los usuarios en las redes sociales (ej. Facebook), favorece la aceptación de las normas de uso y por tanto, de comportamientos de conformidad, a diferencia de los espacios anónimos en la red (Zhao et al., 2008).

Una investigación similar realizada por Krämer y Winter (2008) relacionó la gestión de la impresión personal con los niveles de autoestima, extraversión y autoeficacia, en un grupo de usuarios de una red social alemana en internet. De acuerdo con estos autores, el uso de redes sociales online facilita un mayor control de la conducta de autopresentación que la comunicación cara a cara, y así los usuarios tienen la oportunidad de pensar previamente sobre la información que desean mostrar o elegir aquellas fotos más favorables para su imagen (Krämer \& Winter, 2008). El objetivo de esta investigación fue determinar si los rasgos de personalidad de los usuarios se relacionaban con la manera en que realizaban su autopresentación en la red social. En sus resultados, los usuarios extrovertidos presentaban su perfil de la red de una manera menos restrictiva o conservadora que los usuarios introvertidos; por otra parte, los autores no encontraron relación entre la autoestima y la autopresentación aunque sí entre los niveles de autoeficacia y autopresentación. En esta dirección, aquellos usuarios con altos niveles de autoeficacia utilizaban más información personal en su perfil, mayor número de palabras para describirse y tenían un mayor número de "amigos" (Krämer \& Winter, 2008).
Mehdizadeh (2010) investigó sobre la autopresentación en Facebook y su relación con los niveles de autoestima y los rasgos narcisistas de los usuarios. De acuerdo con esta autora, las redes sociales online han sido etiquetadas como un espacio que expresa el narcisismo, ya que facilitan la construcción de relaciones superficiales, la comunicación emocionalmente indiferente y el control sobre la información que se trasmite sobre sí mismos. Además, las autodescripciones mejoran el self ("yo ideal") y los usuarios reciben feedback público que actúa como un regulador positivo de la autoestima narcisista (Mehdizadeh, 2010). En sus resultados encontró que aquellos usuarios que puntuaron alto en el test sobre narcisismo eran los que tenían mayor frecuencia de acceso a Facebook por día, y también los que pasaban conectados por más tiempo a la red social. Sin embargo, la relación entre la autopromoción en Facebook estuvo correlacionada de forma parcial con la autoestima narcisista: únicamente con el uso de la imagen de perfil, las fotos personales y el "estado" de Facebook. En cuanto a los niveles globales de autoestima de los usuarios, esta se encontró relacionada de manera negativa con la actividad en esta red social; por tanto, aquellos usuarios con baja autoestima tenían mayor frecuencia y tiempo de uso de Facebook que los usuarios con alta autoestima (Mehdizadeh, 2010).

En un estudio anterior, Buffardi y Campbell (2008) estudiaron la relación entre los rasgos narcisistas y el uso de las redes sociales en internet (Facebook). En sus resultados la frecuencia de actividad en el uso de la red (número de amigos y número de comentarios en el "estado" de la red social) estuvo relacionada con los rasgos narcisistas de los usuarios; sin embargo, la autopromoción no se encontró relacionada con el narcisismo. Una investigación similar más reciente señala que la extraversión y el tiempo de conexión online se encontraban relacionadas con rasgos narcisistas en los usuarios, aunque la actualización del estado en la red social no se relacionó con estos rasgos de personalidad (Wang, 2017).

En la actualidad el interés de investigación sobre identidad está en la red social Instagram (por su creciente uso) y de los selfies como modalidad de autopresentación y autopromoción en internet (Deeb-Swihart, Polack, Gilbert \& Essa, 2017; Sheldon \& Bryant, 2016). Los usuarios de esta red suelen ser en su mayoría adolescentes y jóvenes que la usan "por ser cool" (ya que está de moda y es popular), para mantenerse al día sobre sus amigos, mostrar su lado más creativo y poder recibir likes y comentarios, así como una herramienta para documentar sus actividades de viaje u ocio (Sheldon \& Bryant, 2016). Los rasgos narcisistas de los usuarios se muestran en el deseo de ser percibidos de manera positiva y cool, ya que en muchas ocasiones las fotos son manipuladas (usando los diferentes filtros de Instagram) y, además, el tipo de interacción o comunicación en los post suelen ser superficiales (Sheldon \& Bryant, 2016). En cuanto a los selfies, Deeb- Swihart et al. (2017) realizaron un análisis de los diferentes tipos que se publican en Instagram utilizando categorías identitarias. Esto autores encontraron que los jóvenes entre 18 y 35 años son los que más publican selfies y dentro de este grupo las chicas superan a los chicos en número de publicaciones. Las categorías más utilizadas en los selfies son las relacionadas con la apariencia física (por ejemplo, estar a la moda o saludable) y las actividades sociales: estar con amigos, pareja, de cumpleaños, etc. Por tanto, las personas en Instagram "se esfuerzan en parecer felices, saludables y exitosas" (Deeb-Swihart et al., 2017, p. 8). 
Investigaciones sobre las Relaciones Personales en Internet y las Redes Sociales

Las redes sociales en internet se han convertido en un espacio de interrelación para las personas, en el que se sostienen vínculos importantes de amistad, familiares, de pareja y relaciones grupales en general (Bargh \& McKenna, 2004; Kalpidou, Costin \& Morris, 2011; Nabi, Prestin \& So, 2013;). Facebook surgió precisamente para vincular a estudiantes y actualmente es una del redes sociales más utilizadas a nivel mundial (Nabi et al., 2013; Kalpidou et al., 2011). Los estudios iniciales se preocuparon por estudiar estas nuevas tipologías de "amistad" ya que no siempre el número de amigos reflejado en la red social se correspondía con el número de "verdaderos amigos". Por ejemplo, solo entre un 25 y $30 \%$ de las personas en una red social como Facebook son considerados "amigos reales" por los usuarios (Ellison \& Boyd, 2013).

Además, el término "amigo" en una red social indica solo una conexión consensuada entre dos usuarios (implica por lo general recibir "invitaciones", aceptarlas, ignorarlas o rechazarlas) y por tanto no incorpora, necesariamente, el significado psicosocial y sociológico que posee la amistad (Boyd, 2008; Ellison \& Boyd, 2013). Esta situación es ahora más compleja porque las redes sociales permiten seguir a otros usuarios sin que haya, necesariamente una reciprocidad (como Twitter o Lindkedin) lo que amplía la red de relaciones que una persona puede tener en internet (Ellison \& Boyd, 2013). No obstante, los estudios más recientes sostienen que los usuarios de Facebook eligen sus contactos dentro de la red de personas que consideran sus amigos o que les valoran como tal y por tanto son más selectivos que cuando comenzó a utilizarse esta red. Además, el perfil de usuarios de Facebook ha cambiado ya que inicialmente eran más adolescentes y jóvenes y ahora la mayoría de usuarios son jóvenes y adultos lo que hace que se sostengan relaciones de amistad con las personas que realmente se consideran cercanas (Anderson, Fagan, Woodnutt \& Chamorro- Premuzic, 2012; Meng, Martínez, Holmstrom, Chung \& Cox, 2016).

La formación de relaciones de pareja en internet también es un área de investigación que ha crecido en los últimos años gracias a las numerosas páginas web y aplicaciones informáticas dedicadas a este tema (Blackhart, Fitzpatrick \& Williamson, 2014). La mayoría de las investigaciones se han centrado en las formas en que las personas crean su perfil y realizan su presentación en esas redes (auto-presentación), los rasgos de personalidad que influyen en la formación de pareja a través de internet o los factores predisposicionales que predicen el uso de este tipo de redes sociales (Blackhart et al., 2014). Aunque sigue existiendo un porcentaje bajo de perfiles "falsos" o modificados para ser más atractivos, cada vez son más los usuarios de estas redes que incluyen una presentación más cercana a lo que es su identidad actual (Whitty, 2008). Dentro de los rasgos de personalidad que predicen el uso de este tipo de redes sociales están la extroversión o el neuroticismo, ya que ofrece un sentido de identidad y facilita la búsqueda de personas que puedan compartir rasgos y aficiones similares (Clemens, Atkin \& Krishnan, 2015). En cuanto a los factores predisposicionales, la sensibilidad al rechazo interpersonal parece ser uno de los predictores para el uso de redes sociales en la búsqueda de pareja (Blackhart et al., 2014). Sin embargo, los estudios más recientes sugieren que el perfil de las personas que suelen utilizar las páginas web especializadas en búsqueda de pareja y las aplicaciones informáticas (como por ejemplo Tinder), no difieren mucho de la población general que no las utiliza (Gatter \& Hodkinson, 2016).

\section{Investigaciones sobre el Apoyo Social en Internet y las Redes Sociales}

El apoyo social que reciben las personas se ha extendido de forma considerable con el uso de las redes sociales e internet (Ellison, Vitak, Gray \& Lampe, 2014; Meng et al., 2016). Las redes sociales en internet aumentan la percepción de capital social (la habilidad de los individuos o grupos para acceder a los recursos disponibles de su red social) y también de apoyo social, es decir la disponibilidad de apoyo y ayuda en las relaciones interpersonales (Ellison et al., 2014; Heaney \& Israel, 2008).

Distintos grupos sociales se han beneficiado de los chats, foros y comunidades online para dar visibilidad a sus reivindicaciones, sus dificultades, superar barreras y recibir apoyo social, por ejemplo, los grupos estigmatizados o los grupos de apoyo para personas con enfermedades crónicas o graves (Bargh \& McKenna, 2004; Fuente, Herrero \& Gracia, 2010). En la actualidad existen muchos grupos de apoyo social online sobre temas médicos o psicológicos (entre otros), que funcionan activamente. Así, algunas investigaciones reportan beneficios de pertenecer a este tipo de colectivos en pacientes con enfermedades crónicas (Barrera, Glasgow, McKay, Boles \& Feil, 2002; Beléndez \& Suría, 2010) y en personas con problemas de salud mental usuarios de YouTube en los que los pacientes comparten vídeos y responden a comentarios de personas con problemas similares, creando una red de apoyo social (Naslund, Grande, Aschbrenner \& Elwyn, 2014). También, se han observado beneficios en la disminución del estrés en los usuarios de Facebook. Por ejemplo, Nabi et al. (2013) encontraron una relación entre el número de amigos en esa red social y la percepción de apoyo social, lo que contribuía a la disminución del estrés y mayor bienestar psicológico (Nabi et al., 2013). En general, los beneficios de las redes sociales en procesos de salud se resumen en una mejor percepción de control de la enfermedad, acceso a información especializada que reduce la incertidumbre, mejora de las habilidades para tomar decisiones sobre tratamientos y también pueden ayudar a tener una interpretación más positiva sobre la situación (Beléndez \& Suría, 2010; Heaney \& Israel, 2008).

Meng et al. (2016) realizaron una revisión sobre las investigaciones publicadas desde el año 2004 hasta el 2015 sobre apoyo social y redes sociales. Estos autores señalan que Facebook aparece en más del $50 \%$ de investigaciones, seguida de la red Twitter con más del 10\%, lo que hace necesario diversificar los estudios para abarcar las nuevas formas de interacción como Snapchat o Instagram, que suelen atraer a un perfil diferente de Facebook (por lo general, adolescentes). Además, es necesario clarificar lo que se entiende por apoyo social y pasar de una definición estructural (como por ejemplo el número de amigos en la red), a una más funcional (amortiguar los daños que pueda causar el estrés sobre el bienestar del individuo) que se aproxime a la definición más utilizada en investigación (Meng et al., 2016). 
Investigaciones sobre el Bienestar Psicológico en Internet y las Redes Sociales

Un tema importante de investigación es el beneficio que puede tener el uso de las redes sociales en internet para el bienestar psicológico y social. Por ejemplo, Ellison, Steinfield y Lampe (2008) estudiaron la relación entre el uso de Facebook, el aumento del capital social de los usuarios, su nivel de autoestima y satisfacción con la vida. El uso que los usuarios -participantes de este estudio- hacían de Facebook, estaba relacionado con mantener los vínculos ya existentes con su red de amigos, más que para sostener relaciones temporales o efímeras con personas conocidas. Además, aquellos usuarios con un alto nivel de autoestima utilizaban con mayor frecuencia la red social y se sentían más satisfechos. En un estudio similar realizado por los mismos autores, se investigó durante dos años a un grupo de usuarios de Facebook a partir de diversas entrevistas en profundidad y medidas relacionadas con el bienestar psicológico y el incremento del capital social (Steindfield, Ellison \& Lampe, 2008). En este grupo de participantes la red social facilitaba el mantenimiento de las relaciones existentes y permitía la incorporación de nuevas redes sociales creadas a partir del inicio de sus estudios universitarios, especialmente en aquellos que se habían desplazado desde sus lugares de origen para estudiar. En el caso de los usuarios con bajos niveles de autoestima, el uso de Facebook les permitía mejorar su comunicación en la interacción social con los demás y minimizar el miedo al rechazo. De igual manera, tanto el incremento de uso de la red como el aumento del capital social de los usuarios se relacionaron de manera positiva con el bienestar psicológico (Steindfield et al., 2008).

Kalpidou et al. (2011) también estudiaron la relación entre el bienestar (autoestima, ajuste emocional y ajuste social) y el uso de Facebook en un grupo de estudiantes. De acuerdo con sus resultados, a mayor uso de la red social los participantes mostraron mayores niveles de autoestima. Sin embargo, a diferencia de los estudios anteriores, los usuarios frecuentes de la red con baja autoestima mostraron también un buen ajuste emocional y social. En este sentido, el número de amigos en la red estuvo relacionado de manera positiva con el ajuste social de los participantes.

En general, las investigaciones sostienen la hipótesis de la estimulación, es decir, que las redes sociales promueven la interacción social y por tanto contribuyen de manera positiva al bienestar general (Valkenburg \& Peter, 2007). Por ejemplo, en una revisión crítica sobre las investigaciones que relacionan el bienestar o malestar psicológico y el uso de las redes sociales, los autores concluyen que existe una relación positiva entre su uso activo y el bienestar (Verduyn, Ybarra, Résibois, Jonides \& Kross, 2017). Es decir, ser un usuario activo de las redes sociales permite que los individuos cuenten con un capital social y se sientan conectados con otras personas de manera importante, a diferencia de aquellos usuarios más pasivos, que suelen ser espectadores de la vida online de otros y que pueden sentirse mal al establecer comparaciones sociales negativas (Verduyn et al., 2017).

\section{Investigaciones más recientes}

Teniendo en cuenta el uso masivo de las redes sociales, de internet, de los teléfonos inteligentes o smartphones y la aparición de miles de aplicaciones informáticas cada día, es inne- gable que la investigación en psicología seguirá avanzando en esta área.

Uno de los nuevos escenarios de investigación es el big data o la utilización de datos masivos disponibles en internet y el uso de aplicaciones informáticas en los teléfonos inteligentes ( $\mathrm{Ar}$ mayones, Gómez-Zúñiga, Hernández \& Pousada, 2015; Gosling \& Mason, 2014; Harlow \& Oswall, 2016). Como señalan Armayones et al. (2015), lo anterior:

puede llevarnos de una Psicología que a menudo ha visto limitado su alcance por la dificultad de acceso a grandes muestras de individuos en su contexto natural, a una Psicología que disponga de métodos que permitan el acceso a cantidades de información generada en el contexto natural de las personas procedente de muestras de miles de ellas. (p. 22)

Las áreas de investigación en psicología que se pueden beneficiar del uso del big data y las aplicaciones informáticas son variadas, no obstante su uso más frecuente está relacionado con el bienestar, la depresión, el consumo de drogas o las redes sociales, entre otras (Harlow \& Oswall, 2016).

Existen varios estudios sobre las redes sociales que utilizan datos masivos disponibles (big data). Por ejemplo, en Facebook al dar consentimiento para el uso de los datos, los usuarios permiten que puedan analizarse no solo características demográficas sino también las relacionadas con el uso de la red, el tipo de contenido que se comparte (mensajes, fotos, etc.) o los likes que pueden ofrecer información sobre el comportamiento social de los individuos (Gosling \& Mason, 2014). También es posible mostrar cómo el análisis de grandes datos en redes sociales puede ofrecer evaluaciones de personalidad similares a las que se realizan de forma tradicional (Armayones et al., 2015). Otro ejemplo son los análisis que puede hacerse del contenido que se publica en Twitter (Gosling \& Mason, 2014). Para profundizar en este tema puede consultarse el número especial de big data en psicología en la revista Psychological Methods.

En cuanto a los teléfonos inteligentes, el hecho de disponer de una tecnología que ofrece grandes datos y en tiempo real (como en las aplicaciones informáticas), los datos recogidos permiten un análisis del comportamiento inmediato de las personas que ofrecen una relación entre patrones de conducta y diversas variables de resultado como pueden ser los trastornos o el bienestar psicológico (Harlow \& Oswall, 2016). Además, uno de los sectores de investigación que más crece actualmente es el diseño de aplicaciones informáticas específicas para la intervención psicológica (m-Health). Por ejemplo, Dulin y González (2017) utilizaron una aplicación específica en la intervención sobre el consumo adictivo de alcohol para mejorar las estrategias de afrontamiento. También se han utilizado para la intervención en estrés postraumático (Kunh et al., 2017) o en la autorregulación de pacientes con trastorno bipolar (Gliddon, Barnes, Murray \& Michalak, 2017).

De esta forma, tanto el big data como las aplicaciones informáticas son unas herramientas que contribuyen a la mejora de la investigación psicológica al permitir contar con grandes cantidades de datos y que requieren de equipos multidisciplinares que los puedan abordar de manera efectiva (Armayones et al., 2015). De la misma forma, permiten la intervención en tiempo real dirigida a grupos o individuos que necesitan 
atención psicológica, lo que sin duda es una mejora para la eficacia de esta disciplina (Gliddon et al., 2017; Harlow, 2016; Kuhn et al., 2017).

\section{Discusión}

Tanto las redes sociales como otras herramientas de internet han creado espacios nuevos de interrelación entre las personas (con una dinámica de interacción social propia), que han impulsado la investigación psicológica en los últimos 15 años (Gosling \& Mason, 2014). Se ha avanzado desde las investigaciones iniciales acerca de la construcción de la identidad online de los usuarios de la red a los estudios sobre la utilización masiva de los selfies como modalidad de autopresentación y autopromoción, y al creciente uso de redes sociales con un alto componente de exposición personal como Instagram (Deeb-Swihart et al., 2017). Además, las redes sociales son actualmente espacios de interrelación entre las personas en "tiempo real" donde se crean y sostienen importantes vínculos con los demás (Kalpidou et al., 2011). En los últimos años también ha crecido el número de aplicaciones y portales especializados en las búsquedas de parejas que también han abierto nuevas líneas de investigación en psicología (Blackhart et al., 2014).

Adicionalmente, internet y las redes sociales continúan siendo una herramienta de apoyo social para diferentes grupos de personas y se utilizan no solo para dar apoyo informacional sino, especialmente, para ofrecer apoyo emocional en situaciones de dificultad o afrontamiento de eventos vitales estresantes (Ellison et al., 2014; Meng et al., 2016). También se ha vinculado el uso de internet y las redes sociales con el aumento del bienestar psicológico, al sostener que son herramientas que, por sus propias características, promueven la interacción social, la que está vinculada a un mayor aumento del bienestar de los individuos (Verduyn et al., 2017).

Actualmente, los nuevos escenarios de investigación se dirigen hacia la utilización de datos masivos (big data) y las aplicaciones informáticas como herramientas que facilitan la obtención de gran cantidad de datos sobre los individuos, y que permiten, además, realizar intervenciones psicológicas en tiempo real (Armayones et al., 2015; Gliddon et al., 2017; Harlow, 2016; Kuhn et al., 2017).

En este artículo se han presentado solo algunas de las principales líneas de investigación en esta área, lo que requiere de futuros estudios de revisión más detallados que puedan abarcar todos los temas más estudiados dentro de la psicología en internet y las redes sociales. Además, se sugiere seguir atentamente la evolución de los estudios en esta línea ya que por sus propias características (al estar vinculado con las tecnologías) supone un reto de actualización constante para quienes investigan en psicología.

\section{Referencias}

Anderson, B., Fagan, P., Woodnutt, T. \& Chamorro-Premuzic, T. (2012). Facebook Psychology: popular questions answered by research. Psychology of Popular Media Culture, 1(1), 23-37. doi:10.1037/a0026452

Armayones, M., Gómez-Zúñiga, B., Hernández, E. \& Pousada, M. (2015). Big data y Psicología: ¿una oportunidad para el Internet de las personas?. Aloma, Revista de Psicologia, Ciències de IÈducació $i$ de l'Esport, 33(2), 21-29. Recuperado de http://www.revistaaloma.net/index.php/aloma/article/view/266/179

Bargh, J. \& McKenna, K. (2004). The internet and social life. Annual Review of Psychology, 55, 573-590. doi:10.1146/annurev.psych.55.090902.141922

Barrera, M., Glasgow, R., McKay, G., Boles, S. \& Feil, E. (2002). Do Internet-Based Support Interventions Change Perceptions of Social Support?: An Experimental Trial of Approaches for Supporting Diabetes Self-Management. American Journal of Community Psychology, 30(5), 637-654. doi:10.1023/A:1016369114780

Beléndez, M. \& Suría, R. (2010). Apoyo a un "click" de ratón. Los foros de internet para problemas de salud. Acción Psicológica, 7(1), 1-29. doi:10.5944/ap.7.1.202

Blackhart, G., Fitzpatrick, J. \& Williamson, J. (2014). Dispositional factors predicting use of online dating sites and behaviors related to online dating. Computers in Human Behavior, 33, 113-118. doi:10.1016/j. chb.2014.01.022

Boyd, D. (2008). Facebook's privacy trainwreck. Exposure, invasion and social convergence. Convergence: The International Journal of Research into New Media Technologies, 14(1), 13-20. doi:10.1177/1354856507084416

Buffardi, L. \& Campbell, W. (2008). Narcissism and social networking web sites. Personality and Social Psychology Bulletin, 34(10), 1303-1314. doi:10.1177/0146167208320061

Casler, K., Bickel L. \& Hackett, E. (2013). Separate but equal? A comparison of participants and data gathered via Amazon's MTurk, social media, and face-to-face behavioral testing. Computer in Human Behavior, 29(6), 2156-2160. doi:10.1016/j.chb.2013.05.009

Clemens, C., Aktin, D. \& Krishnan, A. (2015). The influence of biological and personality traits on gratifications obtained through online dating websites. Computer in Human Behavior, 49, 120-129. doi:10.1016/j. chb.2014.12.058

Deeb-Swihart, J., Polack, C., Gilbert, E. \& Essa, I. (2017). Selfie-presentation in everyday life: a large-scale characterization of selfie contexts on Instagram. Trabajo presentado en la Eleventh International AAAI Conference on Web and Social Media (ICWSM 
2017), Georgia Institute of Technology, Atlanta. Recuperado de https://aaai.org/ocs/index.php/ ICWSM/ICWSM17/paper/view/15692

Dulin, P. \& González, V. (2017). Smartphone-based, momentary intervention for alcohol cravings amongst individuals with an alcohol use disorder. Psychology of Addictive Behaviors, 31(85), 601-607. doi:10.1037/ adb0000292

Ellison, N. \& Boyd, D. (2013). Sociality through social network sites. En W. Dutton (Ed.), The Oxford handbook of internet studies (pp. 151-172). Oxford: Oxford University Press.

Ellison, N., Steinfield, C. \& Lampe, C. (2007). The benefits of Facebook "friends:" Social capital and college students' use of online social network sites. Journal of Computer-Mediated Communication, 12, 1143-1168. doi:10.1111/j.1083-6101.2007.00367.x/abstract

Ellison, N., Vitak, J., Gray R. \& Lampe, C. (2014). Cultivating social resources on social network sites: Facebook relationship maintenance behaviors and their role in social capital processes. Journal of Computer-Mediated Communication, 19(4), 855-870. doi:10.1111/jcc4.12078/abstract

Fuente, A., Herrero J. \& Gracia, E. (2010). Internet y apoyo social: sociabilidad online y ajuste psicosocial en la sociedad de la información. Acción Psicológica, 7(1), 9-15. doi:10.5944/ap.7.1.201

Gatter, K. \& Hodkinson, K. (2016). On the differences between Tinder versus online dating agencies: questioning a myth. An exploratory study. Journal Cogent Psychology, 3(1). doi:10.1080/23311908.2016.1162414

Gliddon, E., Barnes, S., Murray G. \& Michalak, E. (2017). Online and mobile technologies for self-management in bipolar disorder: a systematic review. Psychiatric Rehabilitation Journal, 40(3), 309-319. doi:10.1037/ prj0000270

Gosling, S. \& Mason, W. (2014). Internet research in Psychology. Annual Review of Psychology, 66(26), 1-26. doi:10.1146/annurev-psych-010814-015321

Harlow, L. \& Oswall, F. (2016). Big data in psychology: Introduction to the special issue. Psychological Methods, 21(4), 447-457. doi:10.1037/met0000120

Heaney, K. \& Israel, B. (2008). Social Network and Social Support. En K. Glanz, B. Rimer \& K. Viswanath (Eds.), Health Behavior and Health Education. Theory, Research and Practice (4th ed., pp.189-210). San Francisco: Jossey-Bass, A Wiley Imprint.

Kalpidou, M., Costin, D. \& Morris, J. (2011). The relationship between Facebook and the well-being of undergraduate college students. Cyberpsychology, Behavior, and Social Networking, 14(4), 183-189. doi:10.1089/cyber.2010.0061
Kosinski, M., Matz, S., Gosling, S., Popov, V. \& Stitwell, D. (2015). Facebook as a research tool for the social sciences: opportunities, challenges, ethical considerations, and practical guidelines. American Psychology, 70(6), 543-556. doi:10.1037/a0039210

Krämer, N. \& Winter, S. (2008). Impression Management 2.0. The relationship of self-esteem, extraversion, self-efficacy, and self-presentation within social networking sites. Journal of Media Psychology, 20(3), 106-116. doi:10.1027/1864-1105.20.3.106

Kunh, E., Kanuri, N., Hoffman, J., Garvert, D., Ruzek, J. \& Taylor, C. (2017). A randomized controlled trial of a smartphone app for posttraumatic stress disorder symptoms. Journal of Consulting and Clinical Psychology, 85(39), 267-273. doi:10.1037/ccp0000163

Lazar, J., Feng, J. \& Hochheiser, H. (2010). Research methods in human-computer interaction. London: Wiley $\mathrm{Pu}-$ blishing.

Mehdizadeh, S. (2010). Self-Presentation 2.0: Narcissism and Self-Esteem on Facebook. Cyberpsychology, Behavior, and Social Networking, 13(4), 357-364. doi:10.1089/cyber.2009.0257

Meng, J., Martínez, L., Holmstrom, A., Chung, M. \& Cox, J. (2016). Research on social networking sites and social support from 2004 to 2005: A narrative review and directions for future research. Cyberpsychology, Behavior and Social Networking, 20(1), 4451. doi:10.1089/cyber.2016.0325

Nabi, R., Prestin A. \& So, J. (2013). Facebook friends with (health) benefits? Exploring social network site use and perceptions of social support, stress, and well-being. Cyberpsychology, Behavior, and Social Networking, 16(10), 721-727. doi:10.1089/cyber.2012.0521

Naslund, J., Grande, S., Aschbrenner K. \& Elwyn, G. (2014). Naturally occurring peer support through social media: the experiences of individuals with severe mental illness using YouTube. Plos One, 9(10), 1-10. doi:10.1371/journal.pone.0110171

Sargis, E., Skitka, L. \& McKeever, W. (2013). The Internet as psychological laboratory revisited: Best practices, challenges, and solutions. En Y. Amichai-Hamburger (Ed.), The social net: The social psychology of the Internet (2nd ed., pp. 253-270). Oxford: Oxford University Press.

Sitka, L. \& Sargis, E. (2006). The internet as psychological laboratory. Annual Review of Psychology, 57, 529555. doi:10.1146/annurev.psych.57.102904.190048

Sitka, L. \& Sargis, E. (2005). Social psychological research and the Internet: the promise and peril of a new methodological frontier. En Y. Amichai-Hamburger (Ed.), The social net human behavior in cyberspace ( $\mathrm{pp}$. 1-25). Oxford: Oxford University Press.

Sheldon, P. \& Bryant, K. (2016). Instagram: motives for its 
use and relationship to narcissism and contextual age. Computers in Human Behavior, 58, 89-97. doi:10.1016/j.chb.2015.12.059

Steindfield, C., Ellison, N. \& Lampe, C. (2008). Social capital, self-esteem, and use of online social network sites: A longitudinal analysis. Journal of Applied Developmental Psychology, 29(6), 434-445. doi:10.1016/j. appdev.2008.07.002

Valkenburg, P. \& Peter, J. (2007). Online communication and adolescent well-being: testing the stimulation versus the displacement hypothesis. Journal of Computer-Mediated Communication, 12(4), 1169-1182. doi:10.1111/j.1083-6101.2007.00368.x/abstract

Verduyn, P., Ybarra, O., Résibois, M., Jonides, J. \& Kross, E. (2017). Do Social Network Sites Enhance or Undermine Subjective Well-Being? A Critical Review. Social Issues and Policy Review, 11(1), 274-302. doi:10.1111/sipr.12033/abstract

Wang, D. (2017). A study of the relationship between narcissism, extraversion, drive for entertainment, and narcissistic behavior on social networking sites. Computers in Human Behavior, 66, 138-148. doi:10.1016/j.chb.2016.09.036

Whitty, M. (2008). Revealing the 'real' me, searching for the 'actual' you: Presentations of self on an internet dating site. Computer in Human Behavior, 24(4), 1707-1723. doi:10.1016/j.chb.2007.07.002

Wolfe, C. (2017). Twenty years of Internet-based research al SCiP: a discussion of surviving concepts and new methodologies. Behavior Research Methods, 49(5), 1615-1620. doi:10.3758/s13428-017-0858-X

Zhao, S., Grasmuck, S. \& Martin, J. (2008). Identity construction on Facebook: Digital empowerment in anchored relationships. Computers in Human Behavior, 24, 1816-1836. doi:10.1016/j.chb.2008.02.012 